\title{
La cadena de frío en la industria farmacéutica: Del fabricante al paciente
}

\author{
Úrsula Vértiz Combe \\ Salud y Logística S.A. (Salog) \\ Ingeniería Industrial n. ${ }^{\circ} 29,2011$, ISSN 1025-9929, pp. 11-34 \\ Recibido: 25 de febrero del 2011 / Aprobado: 15 de abril del 2011
}

REsumen: El desarrollo de la industria farmacéutica está orientado a la medicina preventiva, con la producción de medicamentos biotecnológicos que requieren una cadena de frío de $2^{\circ} \mathrm{C}$ a $8^{\circ} \mathrm{C}$. En el presente artículo se propone un modelo de gestión de la cadena de frío que toma en cuenta aspectos técnicos de infraestructura, procesos, análisis de riesgos y entrenamiento, aspectos clave para el desarrollo de una adecuada logística, que garantice la calidad de los productos, manteniendo las mismas condiciones a lo largo de todo el proceso, desde que estos salen de la planta de manufactura hasta que llegan al paciente.

Palabras clave: productos farmacéuticos / cadena de frío / análisis de riesgo / diseño de embalajes / programa de capacitación

\section{The cold chain in the pharmaceutical industry: From the manufacturer to the patient}

\begin{abstract}
Pharmaceutical industry is focused on preventive medicine, using biotechnology in manufacturing processes, and as a consequence the products require cold chain conditions between $2^{\circ} \mathrm{C}$ to $8^{\circ} \mathrm{C}$. This paper explore cold chain management system, based on infrastructure, processes, risk analysis, training program, keywords to assurance high quality, and maintaining cold chain conditions from manufacturing plant to the patient.
\end{abstract}

Keywords: pharmaceuticals products / cold chain system / risk analysis / packaging design / training program 


\section{INTRODUCCIÓN}

Pobreza, desnutrición, miseria son denominadores comunes en la Comunidad Andina, donde la empresa privada y el Estado juegan un rol preponderante para mejorar la calidad de vida de las personas, especialmente de los niños y la población indefensa.

En este contexto surge como una necesidad el desarrollo de la medicina preventiva, básicamente conformada por productos biotecnológicos altamente especializados, como las vacunas.

La correcta administración de los recursos humanos y técnicos de la cadena de frío son claves para garantizar que los productos altamente especializados lleguen al destino final y cumplan los estándares para los cuales fueron diseñados, con el fin de ser eficientes en la prevención de las enfermedades que aquejan a nuestra sociedad.

Por ello, en las siguientes páginas presentaremos las tendencias mundiales en la fabricación de productos farmacéuticos así como las diferentes etapas de la cadena de suministro en frío, conocida simplemente como "cadena de frío", entendida como un sistema de gestión integral que comprende buenas prácticas y análisis de riesgos.

\section{ANTECEDENTES}

Las formas farmacéuticas tradicionales: sólidos, líquidos o ungüentos, entre otros, son aquellas de fabricación para tratamientos curativos. En el mundo globalizado las compañías farmacéuticas investigan incansablemente para poder alcanzar logros significativos en la prevención de enfermedades, con la finalidad de que los seres humanos puedan acceder a mejores condiciones de vida y salud.

Los ingenieros industriales no son ajenos a ese proceso, y en ellos ha recaído el diseño de los sistemas de suministro, puntos de reorden y logística especializada en este tipo de productos, en Estados Unidos, Europa, Asia y Latinoamérica, que trae consigo una serie de cuidados y requerimientos.

A continuación se presentan algunos datos relevantes:

- Entre 1999 y 2003 el mercado de productos biotecnológicos creció un $21 \%$ versus un crecimiento de $11 \%$ de los productos farmacéuticos tradicionales. 
- 17 productos de biotecnología fueron aprobados a mediados de abril del 2008 versus 9 que se aprobaron en el primer trimestre del 2007.

- Cada una de las 7 compañías que maneja biotecnología creció sustancialmente desde el 2006 y en el primer trimestre del 2008 sobrepasó el total vendido en el 2007.

- El 20\% de los productos farmacéuticos más vendidos son sensibles a la temperatura.

- Más de 130 billones de dólares del mercado farmacéutico están representados por productos sensibles a la temperatura.

- Cerca del $100 \%$ de las vacunas y $68 \%$ de los productos comercializados por compañías de biotecnología necesitan ser almacenados y transportados en un rango de temperatura de $2^{\circ} \mathrm{C}$ a $8^{\circ} \mathrm{C}$.

\section{DEFINICIÓN DE CADENA DE FRÍO}

Es un conjunto de elementos y procedimientos necesarios para el manejo, la conservación, el almacenamiento y la distribución de productos dentro de un rango de temperaturas específicas, que garantiza su calidad desde la salida del laboratorio fabricante hasta su administración al paciente.

Las familias de temperatura de almacenaje más utilizadas son:

- Temperatura ambiente (zonas climáticas).

- Fresco: $\quad 8^{\circ} \mathrm{C}$ a $15^{\circ} \mathrm{C}$.

- Refrigerado: $\quad 2^{\circ} \mathrm{C}$ a $8^{\circ} \mathrm{C}$.

- No congelar: $\quad>0^{\circ} \mathrm{C}$.

- Congelado: $\quad<-20^{\circ} \mathrm{C}$.

- Congelación criogénica: -70 o $-180^{\circ} \mathrm{C}$.

\section{PROCESO DE CADENA DE FRÍO}

En la figura 1 se observa el sistema de cadena de frío con una visión por procesos, y se describen los puntos clave de cada proceso, reflejando las buenas prácticas. Se toma en cuenta un almacenamiento refrigerado para los productos de $2^{\circ} \mathrm{C}$ a $8^{\circ} \mathrm{C}$.

Los recuadros verdes representan el flujo logístico, el cual se soporta en los procesos operativos, la infraestructura, el sistema de gestión de calidad y control de procesos, el análisis de riesgos y el diseño del sistema. 


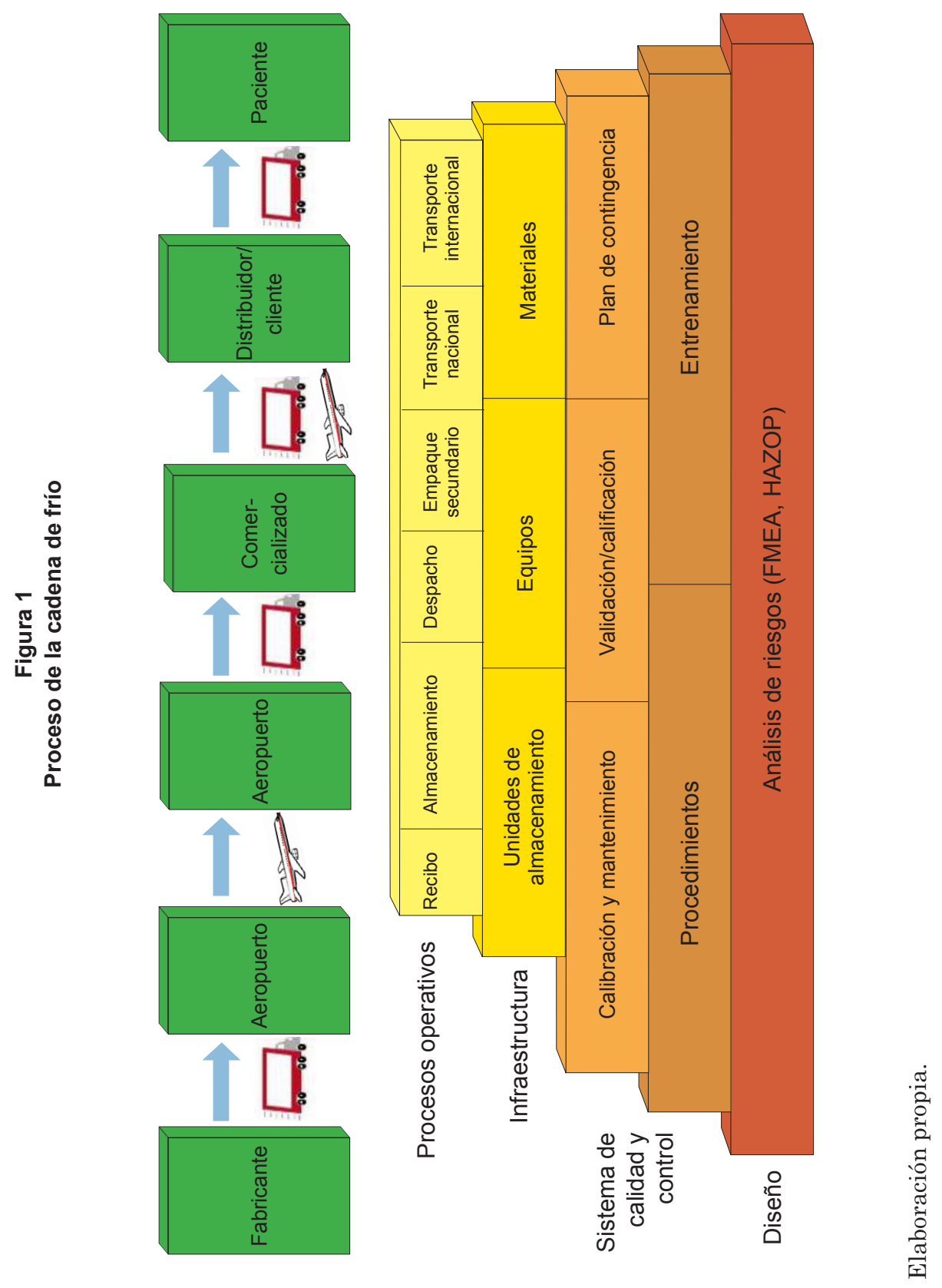




\section{PROCESOS OPERATIVOS}

\subsection{Recepción de mercadería (Recibo)}

La recepción de la mercadería consiste en el proceso de recibo, control y entrega de los productos para la cámara fría. En este caso el producto puede provenir de la aduana y llegar por un proceso de importación, de otro almacén como transferencia, ser un producto en tránsito, o ser su destino final.

El tiempo de ejecución de este proceso es clave para asegurar que los productos ingresen al almacenaje refrigerado lo antes posible; la principal recomendación es que se entrene al personal para que considere como urgente toda recepción de productos que requieran refrigeración. Los puntos identificados en este proceso que se deben tener en cuenta son:

- Notificación de llegada.- Diseñar un sistema que permita a los responsables de los almacenes dar aviso de qué productos de cadena de frío van a ingresar, y darles prioridad de ingreso a los vehículos.

- Inspección de vehículos.- Asegurar que los vehículos lleguen en las condiciones acordes con las buenas prácticas de transporte: limpios, con control de temperatura, furgones cerrados y con los precintos de seguridad correspondientes. Se debe llevar un registro escrito de esta inspección.

- Desmontaje de las cajas.- Contar con un procedimiento operativo para el desmontaje de las cajas refrigeradas, con un método adecuado.

- Verificación de la temperatura del producto.- Retirar con sumo cuidado los registradores electrónicos de temperatura de las cajas de embalaje con un orden correlativo, y realizar la lectura de la temperatura mientras el producto es ingresado en el área de cuarentena de la cámara fría.

- Ubicación en el cuarto frío-. Mientras se procede con la lectura de la temperatura y para realizar la inspección física del ingreso de mercadería, ubicar los productos en un área de cuarentena (almacén de tránsito para inspección) hasta que se pueda liberar el producto.

- Documentar tiempos.- Contar con un sistema electrónico o manual para el control de los tiempos de ejecución y compararlos con un tiempo estándar proyectado para este proceso. 


\subsection{Almacenamiento}

Es recomendable que dentro de la cámara fría se utilicen sistemas manuales y automatizados de almacenamiento, como es el caso de la radiofrecuencia.

$\mathrm{Al}$ respecto, se recomienda establecer lo siguiente:

- Áreas separadas e identificadas.- Acorde con lo estipulado en las Buenas Prácticas de Almacenamiento, reglamentadas por la Dirección General de Medicamentos, Insumos y Drogas (Digemid) y las Good Manufacturing Practices (GMP) de la Food and Drug Administration (FDA), que disponen que todo almacén, con temperatura controlada o sin ella, cuente con las siguientes áreas identificadas y segregadas:

- Cuarentena.- Espacio donde se almacenan los productos farmacéuticos una vez recibidos, para su muestreo y posterior aprobación o liberación por parte de la Unidad de Calidad respectiva, que se encarga de aprobar el producto para que pase al área de Libre Disponibilidad y salga a la venta, la comercialización o la distribución, según corresponda. En el caso puntual de la cadena de frío se recomienda no solo tener letreros de identificación y un área segregada y separada, sino que el área de cuarentena dentro de la cámara debe estar en una zona cerrada con rejillas, para evitar la confusión de operación y la contaminación cruzada.

- Libre disponibilidad.- Área de almacenaje donde llegan los productos aprobados para su venta, comercialización o distribución, de acuerdo con los requerimientos.

- Devoluciones.- Área identificada donde van los productos devueltos. Se recomienda, al igual que en el caso de Cuarentena, que de preferencia sea cerrada. Aquí la Unidad de Calidad determina si el producto está todavía apto para volver al área de Libre Disponibilidad o si se envía al Área de Rechazos, para su posterior destrucción conforme a ley.

- Controlados.- Productos cuyos principios activos están conformados por una o más sustancias controladas por el Decreto 023 de Digemid, dado que estos productos se venden solo con receta médica retenida y deben permanecer bajo llave. 
- Identificación de ubicaciones.- Para un mejor almacenamiento y control se recomienda dividir la estantería interior con ubicaciones que permitan que, mediante un sistema electrónico, semiautomático o manual, se puedan identificar fácilmente las medicinas.

- Almacenamiento de los materiales de embalaje y refrigerantes.- Contar con un área separada para el almacenamiento de los materiales de embalaje y un área de congelado, congelador o cámara de congelado, para su correcto almacenaje.

- Control de acceso al cuarto frío.- Dado que los productos biotecnológicos son de alto costo, por seguridad se recomienda la restricción de acceso solo al personal debidamente autorizado.

- Ubicación en contenedores que mantengan la integridad y calidad, sin contacto directo con el piso para permitir limpieza, mantenimiento e inspección de rutina.

- Contar con luz de emergencia, apta para bajas temperaturas dentro del cuarto frío, para los casos de corte de energía.

- Condiciones ambientales aseadas y fáciles de limpiar, acordes con las Buenas Prácticas de Almacenamiento.

- Software de control y monitoreo de equipos sobre la base de la norma CFR21 de la FDA, esto es sistema de alarmas para prevención de eventos críticos tales como: alarma sonora, alarma conectada a la vigilancia y equipo técnico responsable, entre otras.

En la figura 2 se observa el interior de una cámara de frío para almacenamiento de vacunas y productos de biotecnología, con cinco niveles de almacenamiento en racks. Se pueden apreciar también tres niveles esquemáticos que representan las capas de frío dentro de la cámara; las figuras redondas indican dónde deben ir los controles de temperatura para monitoreo permanente.

La norma técnica CFR21 recomienda que el monitoreo de la temperatura se realice en los puntos determinados como críticos al momento de validar el equipo:

- Cerca de la puerta de ingreso, por donde se pierde eficiencia del sistema cada vez que se abre.

- Cerca del equipo evaporador y en los extremos, para monitorear si el frío está circulando adecuadamente. 
Figura 2

Almacenamiento en cámara refrigerada

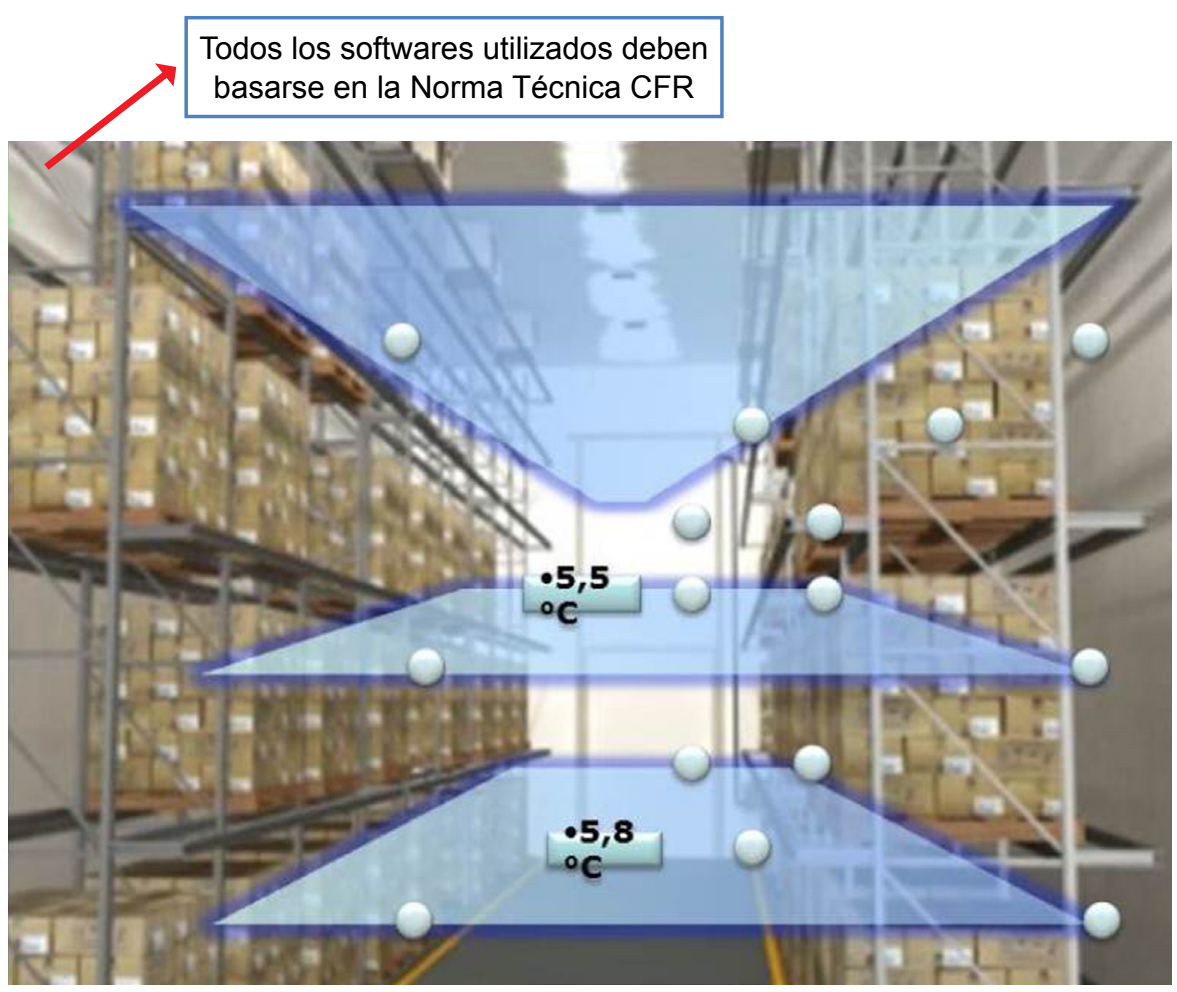

Elaboración propia.

\subsection{Distribución (despacho y empaque)}

Para el despacho y la distribución de productos refrigerados entre $2^{\circ} \mathrm{C}$ y $8^{\circ} \mathrm{C}$ existe una serie de elementos que conforman su embalaje: cajas térmicas de poliuretano expandido (EPS), comúnmente denominadas tecnopores; paños absorbentes y geles refrigerantes o similares, denominados elementos fríos, que permiten desarrollar un microclima dentro del empaque, con el fin de que garanticen el mantenimiento de la temperatura adecuada en los embalajes validados, los procedimientos de embalaje estandarizado, los materiales de distribución aprobados por el Área de Calidad y en la verificación de las condiciones en el vehículo y el transporte. 
En la figura 5 se muestran las bolsas de gel pack, que forman parte de los embalajes; y la figura 6 ejemplifica la manera como una caja de embalaje refrigerado está expuesto a condiciones externas de temperatura (cálidas y frías) durante el proceso de distribución.

Para lograr las condiciones internas ideales se requiere de un estudio previo, un diseño de embalaje y pruebas de calificación de diseño que van de la mano con el desarrollo del perfil térmico de la ruta por donde el producto circulará.

Para garantizar adecuadamente la distribución se recomienda lo siguiente:

- Validar las condiciones de embalaje de acuerdo con el medio de transporte empleado para la distribución, el destino de los productos y la ruta a utilizar.

- Los embalajes, según su validación, pueden contener elementos refrigerados o elementos congelados que sean aislantes.

- Tanto para los elementos congelados como para los elementos refrigerados, se debe determinar un tiempo, hasta que alcancen la temperatura requerida según los estudios realizados.

- El montaje de los embalajes debe estar soportado por un procedimiento estandarizado y documentado en una lista de chequeo.

- Los materiales usados para la distribución deben ser aprobados por la Unidad de Calidad y almacenados bajo condiciones ambientales adecuadas.

- La colocación de las cajas en el vehículo de transporte y las condiciones de este deben ser documentadas en una lista de chequeo del transporte.

\subsubsection{Transporte}

Para el transporte de los productos de la cadena de frío, cualquiera que sea la vía seleccionada, se deben considerar vehículos limpios, que cuenten con programas de mantenimiento preventivo.

En caso de que el producto no esté embalado para la conservación de la cadena de frío (en cajas frías o neveras portátiles), los vehículos deben estar acondicionados con materiales aislantes o equipos de refrigeración. 
Se enumeran a continuación algunos aspectos relativos al transporte:

- El transporte debe ser realizado de acuerdo con el estudio de validación.

- Se debe seleccionar el medio de transporte de acuerdo con el estudio de validación. La calificación de desempeño de los embalajes se efectúa en las rutas definidas como críticas.

- Monitorear y registrar la temperatura durante el proceso de transporte en caso de no tener validado un proceso de despacho en neveras o cajas frías.

- Disponer de un plan de contingencia para aplicar durante el transporte en caso de emergencia.

- Si el tiempo de transporte excede el tiempo validado para los embalajes y no se cuenta con registros de la temperatura alcanzada, el producto debe ser rechazado y devuelto al fabricante para su proceso de destrucción. Si se cuenta con los registros, se contactará con el fabricante para que establezca la disposición del producto basado en los registros.

- Registrar la información relacionada con el envío, como la fecha y la hora de salida, el destino (dirección, nombre de la persona de contacto y teléfono), la temperatura de salida, el nombre del producto, el número de lote, entre otros.

- Notificar oportunamente el envío para asegurar el recibo por parte del destinatario y que este tenga claros los cuidados que debe tener para conservar la calidad del producto.

- Alternativa con vehículo refrigerado.

- El contenedor refrigerado debe estar calificado. Los vehículos que exceden los 4 pies suelen presentar áreas frías y calientes, que deben ser identificadas en su calificación. De ser necesario, y según la zona geográfica, se considerará un perfil para invierno y otro para verano.

- Instalar cortinas plásticas de tiras en las puertas de ingreso al contenedor refrigerado.

- El contenedor debe estar limpio y en buenas condiciones de funcionamiento, con un plan de mantenimiento periódico. Estas condiciones serán verificadas antes de que reciba la carga y quedarán documentadas. 
- Tanto el conductor como el auxiliar deben estar entrenados para actuar en caso de falla de los equipos para el mantenimiento de la cadena de frío. Contarán con un procedimiento a seguir en caso de emergencia.

- Antes de cargar el vehículo, este debe preenfriarse hasta lograr la temperatura requerida.

- Durante el cargue y descargue, la puerta se mantendrá abierta el menor tiempo posible.

\subsubsection{Transporte internacional}

Durante el transporte de productos suele presentarse una serie de riesgos, como múltiples manipulaciones, cupos llenos en las rutas validadas, trasbordos, procesos de nacionalización, tiempos de viaje y perfiles de temperatura, pérdidas y daños, y falla de los equipos de refrigeración.

Con el propósito de evitar tales riesgos se debe establecer un plan de contingencia para cada uno de estos, implementar mecanismos de monitoreo de temperatura durante el transporte, informar al destinatario los datos del envío y tener la habilidad de hacer cambios y correcciones durante el viaje.

\section{INFRAESTRUCTURA}

\subsection{Unidades de almacenamiento: Cámara fría o cuarto frío}

Los cuartos fríos son recintos aislados, alejados de fuentes de calor, construidos con un sistema de refrigeración y con un control de temperatura interior que les permite conservar la temperatura de almacenamiento dentro del rango establecido.

Detalles a tener en cuenta:

- La panelería debe estar aprobada y certificada por compañías aseguradoras.

- El cuarto debe estar calificado (IQ, OQ, PQ).

- Se debe tener un logbook para el cuarto frío, en donde se registran todos los eventos relacionados con este (mantenimientos, fallas, activación de alarmas, etcétera). 
- Los equipos asociados deben ser calibrados periódicamente.

- Posición u orientación final del cuarto frío dentro de las instalaciones, de modo que se facilite la manipulación del producto y este no se vea afectado por condiciones ambientales como el polvo, la exposición directa a los rayos del sol, etcétera.

- Instalar cortinas plásticas de tiras en las puertas de ingreso al cuarto frío.

- Iluminación adecuada para una fácil lectura e inspección de los productos. Las lámparas utilizadas deben generar el menor calor posible o contar con un protector aislante de temperatura. Es recomendable instalar lámparas de emergencia en el interior del cuarto.

- Ventilación adecuada para tener una correcta circulación de aire y evitar olores contaminantes.

- Panel de detección y control de incendios dentro del cuarto frío con estación de manejo 24/7.

- Contar con unidades de respaldo validadas del generador de frío en el caso de que la unidad principal falle.

- Contemplar sistemas de alarmas sonoras y visuales, locales y remotas (reporte vía celular, correo electrónico, software, etcétera) para detectar salidas del rango de temperatura o fallas en el funcionamiento de algunos de los componentes del sistema. Estas alarmas deben contar idealmente con una fuente alterna de energía para que en caso de corte del suministro principal de energía, se puedan accionar.

- Las alarmas deben contemplar no solo temas relacionados con las variaciones de temperatura, sino también en los siguientes casos:

- Alarma en el caso de que una persona quede atrapada dentro de la cámara de frío.

- Alarma de puerta abierta, para evitar que el frío se pierda al mantenerse el contacto con el medio ambiente por periodos largos de tiempo.

- Alarma de señal eléctrica de las conexiones del equipamiento, para que si el equipo de refrigeración falla, se pueda alternar con un equipo backup.

- Instalar registradores continuos de temperatura (24 horas/7 días) en lugares de fácil acceso para el monitoreo de la temperatura del cuarto. Es conveniente contar con sistemas de registro que estén conectados a fuentes alternas de energía, para que en el caso de 
corte del suministro principal de energía, se continúe con el registro de datos.

- Posición, acceso y seguridad del panel de control, el equipo de monitoreo y las alarmas, de manera que se puedan manipular fácilmente pero que no queden al libre ingreso de personal no autorizado, para lo cual deben contar con una protección física o una contraseña para el controlador. Se recomienda cerrar con candado o dispositivos de control electrónicos el acceso a llaves eléctricas y al equipo para regulación de temperatura, así como procurar un registro de acceso a las cámaras del personal.

- Organizar todos los equipos de refrigeración, como compresores, condensadores y tableros eléctricos en un cuarto técnico definido con control de acceso pero, al mismo tiempo, de fácil acceso para el mantenimiento de los equipos.

- Disponer de un inventario de repuestos adecuado y un plan de mantenimiento predictivo y preventivo.

- Tener diseñado un plan de mantenimiento preventivo para el cuarto frío.

- Contar con UPS (uninterruptible power supply) o fuente de poder ininterrumpible para mantener la energía de los controladores del equipo mientras funciona el grupo electrógeno, y garantizar continuidad al $100 \%$ de cada elemento que compone el equipamiento del sistema de frío.

\subsection{Sistemas de monitoreo}

Para un correcto monitoreo, en la cámara fría se deben instalar sistemas de alarmas:

- Alarma de temperatura alta.

- Alarma de temperatura Hi/Hi.

- Alarma de temperatura baja.

- Alarma de temperatura Lo/Lo.

- Alarma de pérdida de energía.

- Alarma de pérdida de comunicación.

- Alarma de hombre atrapado.

- Alarma de puerta abierta. 
El registro de estas alarmas debe ser reunido y retenido para la revisión en formulario de papel o, de preferencia, electrónico. La Unidad de Calidad será la encargada de revisarlo.

En cuanto a las termocuplas (sensores de temperatura), estas se deben ubicar en los lugares definidos por la validación del espacio.

\subsection{Almacenamiento de elementos fríos}

Se definen como elementos fríos aquellos geles, pilas de agua, ice sponge y otros elementos que permiten conservar los productos refrigerados en las cajas térmicas que se diseñan para el transporte.

Puntos clave para su almacenamiento:

- Planes de mantenimiento preventivo.

- Equipos calificados (IQ, OQ y PQ [calificación de instalación, operación y desempeño]).

- Identificación de equipos.

- Especificaciones de usuario para cámara de frío o cuarto de congelado de geles.

- Capacidad máxima de almacenamiento para que el aire pueda circular adecuadamente.

- Número de sensores de temperatura adecuados para el control.

- Tiempo de almacenamiento de los refrigerantes y tiempo de climatización para no afectar los productos.

- Rango de temperatura adecuada. Ejemplo: $-15^{\circ} \mathrm{C}$ a $-25^{\circ} \mathrm{C}$.

- Sistema de monitoreo y alarma para garantizar las condiciones de temperatura.

- Manual del equipo.

- Implementos de seguridad. 


\section{Figura 3}

Cámara de congelado de $-20^{\circ} \mathrm{C}$ a $-25^{\circ} \mathrm{C}$ para elementos fríos

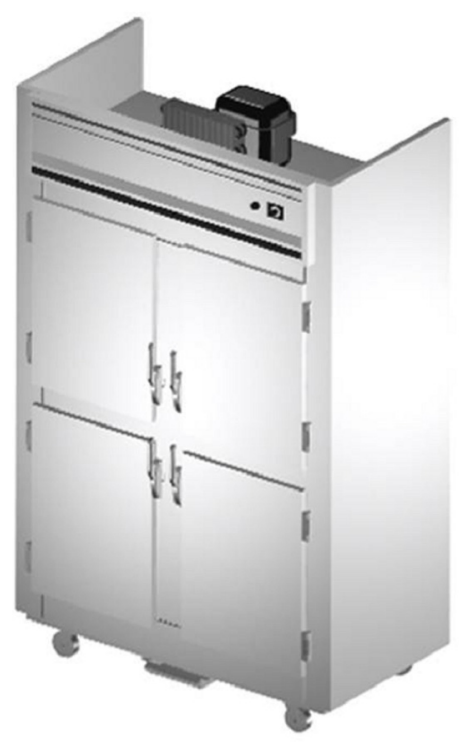

Elaboración propia.

La figura 4 muestra una cámara de congelado de $-20^{\circ} \mathrm{C}$ a $-25^{\circ} \mathrm{C}$ para elementos fríos; al respecto, caben las siguientes recomendaciones:

- Tener línea a grupo electrógeno, al igual que la los productos farmacéuticos estos elementos que los acompañan y sirven para su adecuado transporte deben ser estrictamente cuidados.

- Contar con alarmas sonoras y visuales.

- Contar con botón de emergencia para el caso de que una persona quede atrapada.

- Seguir los lineamientos para cámara refrigerada en cuanto a tipo de alarmas y condiciones de seguridad.

\section{4 Área de acondicionados (precámara recomendada)}

Los productos de cadena de frío requieren un área con temperatura controlada para empacarlos adecuadamente y acondicionarlos para su correcta distribución. Así, las condiciones controladas de temperatura 
deben ser de entre $10^{\circ} \mathrm{C}$ y $12^{\circ} \mathrm{C}$; se requiere documentar el tiempo total cuando los productos son retirados del cuarto frío y contar con procedimientos documentados.

\subsection{Materiales: Empaque y embalaje}

Los empaques pueden ser de cuatro tipos: cajas térmicas, que pueden ser de EPS (poliuretano expandido) o de poliuretano; refrigerantes, que pueden ser geles o ice pack; aislantes, y monitores de temperatura.

En lo que respecta a los embalajes, se deben tener en cuenta ciertas especificaciones técnicas, como la configuración de los geles; los embalajes calificados (DQ, OQ y PQ); el no contacto directo de geles congelados con el producto; el instructivo, con personal entrenado; el tiempo para el alistamiento y embalaje de productos en el área asignada; la temperatura y el tiempo de almacenamiento de los refrigerantes; la lista de verificación y los sensores de temperatura.

Figura 4

Cajas térmicas

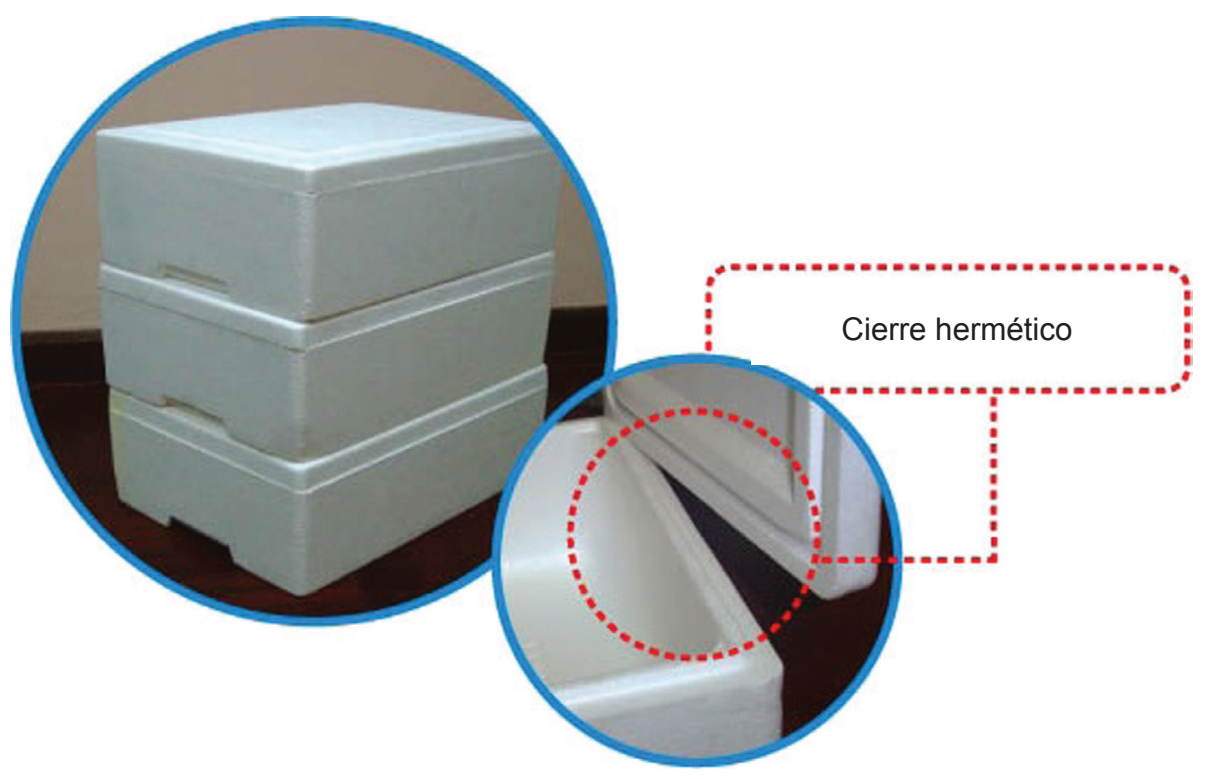

Fuente: Productos Tippic S.A.C. (2011). 
Figura 5

Bolsas de gel pack

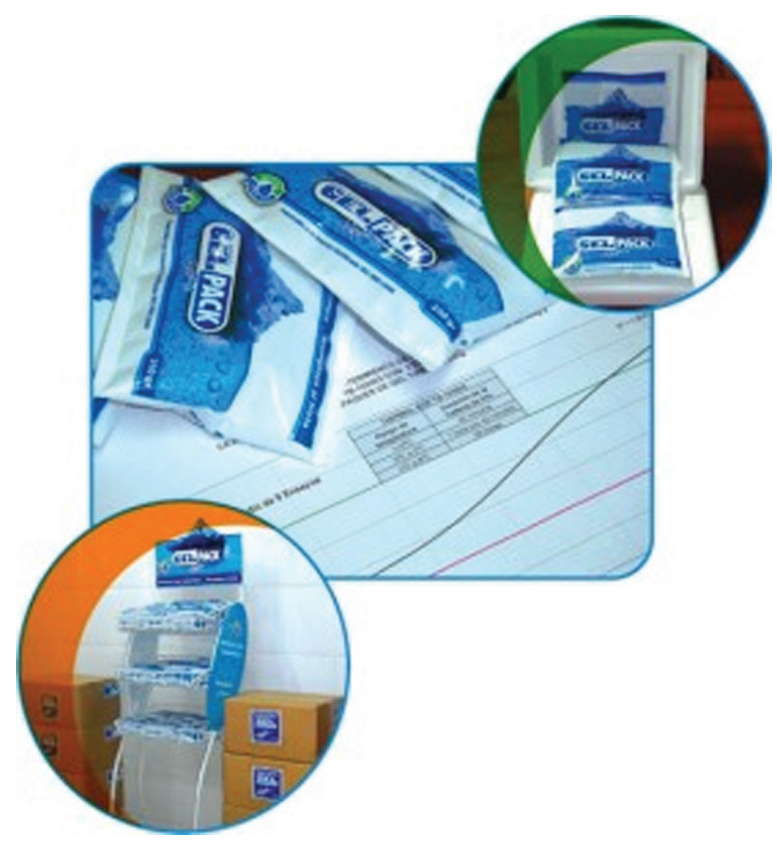

Fuente: Productos Tippic S.A.C. (2011).

Antes de realizar los trabajos de distribución y como buena práctica se recomienda el desarrollo de un perfil de temperatura que permita identificar los puntos críticos para realizar la validación de rutas.

\section{SEGURIDAD Y SALUD OCUPACIONAL EN LA MANIPULACIÓN DE PRODUCTOS REFRIGERADOS}

Como parte del proceso de manipulación de productos en rangos de temperaturas especiales, es muy importante el uso de implementos de seguridad que garanticen el adecuado manejo de los productos y, sobre todo, el cuidado de las personas que están sujetas constantemente a cambios bruscos de temperaturas.

Entre estos implementos se cuentan: guantes, pantalón térmico, casaca térmica y mascarillas que estén de acuerdo con el tipo de producto. 


\section{PERFIL TÉRMICO DE TEMPERATURA}

Con el objetivo de realizar los estudios de distribución se diseña un perfil de temperatura a lo largo de todo el proceso logístico. Se efectúa un estimado de las regiones geográficas y las temperaturas de los lugares por donde va a transitar el producto, buscando identificar los puntos críticos, es decir aquellos donde la temperatura tiende a bajar a $0^{\circ} \mathrm{C}$ o a superar los $25^{\circ} \mathrm{C}$.

Una vez identificadas las temperaturas de las diferentes zonas, como se observa en la figura 6 , se define si se trabajará con un diseño que lleva a un perfil de verano, es decir las condiciones ambientales por encima de $\operatorname{los} 25^{\circ} \mathrm{C}$, o a un perfil de invierno, es decir condiciones ambientales por debajo de $15^{\circ} \mathrm{C}$.

En el caso del Perú tenemos casos extremos. En Piura, por ejemplo, según el Weather Channel, la temperatura máxima es de $34^{\circ} \mathrm{C}$ y la mínima de $17^{\circ} \mathrm{C}$; en cambio, en Puno las temperaturas tienen rangos variables y dependen incluso de la hora, y pueden ir de $-15^{\circ} \mathrm{C} \mathrm{a} 17^{\circ} \mathrm{C}$ en la misma época del año.

Estudiar los climas y tener mapas de distribución actualizados permite, pues, un manejo de esta variable para garantizar que el aislamiento térmico dentro de la caja de embalaje sea el adecuado.

En la figura 6 se aprecia el desenvolvimiento de un perfil térmico desde que sale de la fábrica hasta que llega al destino final.

\section{PLAN DE CONTINGENCIA}

Es necesario contar con una adecuada infraestructura, procedimientos e instructivos de trabajo de soporte, alta tecnología, equipamiento backup y sistemas electrónicos de monitoreo. Pero estos no serán suficientes si no se cuenta con un adecuado plan de contingencia, difundido, comunicado y conocido por todo el personal.

- El Área de Logística requiere contar con procedimientos escritos que describan los planes de contingencia. Todo el personal de las áreas de Logística, Mantenimiento y Seguridad deben estar capacitados en la aplicación de los procedimientos.

- Los planes de contingencia deben basarse en análisis de riesgos FMEA o HAZOP, que tendrán que cubrir todos los aspectos rela- 
Figura 6

Perfil térmico desde la salida de la fábrica

Rutas de estudio: Perú-Bolivia

(Lima-Cusco-Puno-Desagüadero)

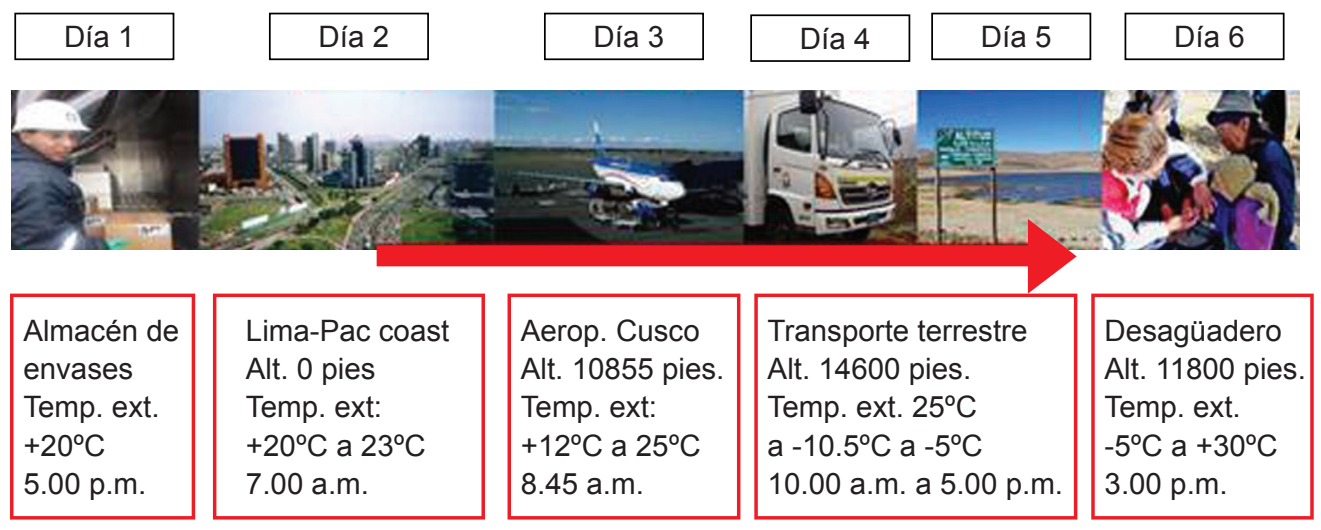
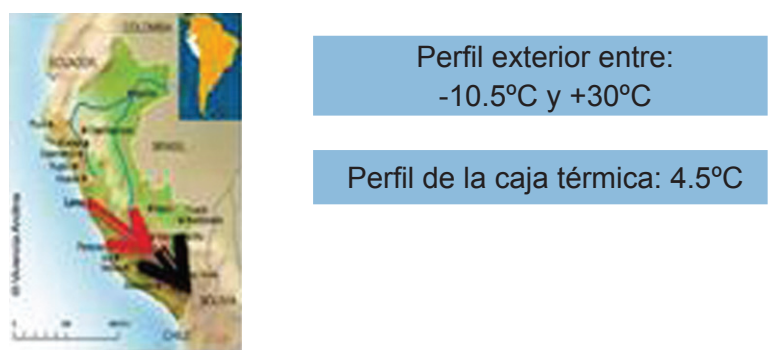

Perfil de la caja térmica: $4.5^{\circ} \mathrm{C}$

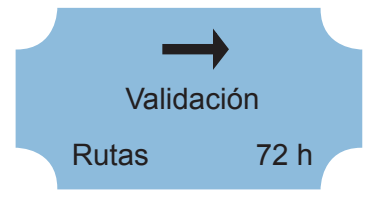

Elaboración propia.

cionados con los equipos, procedimientos, operaciones, seguridad y medioambiente.

- Los planes de contingencia se ejecutarán en los casos en que una situación determinada pueda afectar las condiciones de temperatura de almacenamiento. Ese evento debe ser documentado y la Unidad de Calidad investigará para evaluar la integridad del producto y determinar su disposición final.

- La frecuencia del plan de contingencia depende del riesgo asociado a la complejidad, criticidad e histórico de cambios en los sistemas de cadena de frío. Como mínimo se debe realizar una prueba completa al año. 
- Es necesario que todo el personal sea consciente del plan y de su contenido, y particularmente de sus propios deberes y responsabilidades. La capacitación abarcará al personal operacional, al de liderazgo y al de seguridad.

\section{IMPACTO EN LOS COSTOS DE LA EMPRESA}

De la misma manera que los costos asociados a la calidad son considerados una inversión, en el caso de los costos de transporte para la cadena de frío se sugiere que se les incluya igualmente como parte de la inversión, que garantiza:

- Mantener las propiedades químicas de los productos.

- Cuidar de productos de alto valor monetario.

- Controlar y realizar el seguimiento de una medicina de calidad, con el fin de que la efectividad del fármaco ayude a prevenir enfermedades.

Dado el marco indicado, es conveniente tener en cuenta algunos ratios para la distribución de productos farmacéuticos en el Perú:

- Material de embalaje: 5\% del valor del precio referencial del producto.

- Transporte refrigerado: Aproximadamente US\$450 diarios, con unidades adecuadamente acondicionadas, calificadas, con GPS para monitoreo no solo de rutas sino de temperaturas y control a distancia.

En el cuadro 1 se puede apreciar un ejemplo del costo de embalaje para el envío de Lima a Cajamarca de 800 unidades de vacunas, cuyo valor comercial es de aproximadamente US $\$ 80$.

El costo del transporte es el convencional del envío aéreo, y dado el diseño del embalaje el producto no requiere el uso de condiciones especiales externas; sin embargo, como producto del diseño y la validación hay una serie de materiales y equipos necesarios para la conservación de temperatura. 


\section{Cuadro 1}

Costos de embalaje de una caja por 100 unidades

\begin{tabular}{|c|c|c|c|c|}
\hline Descripción & Cantidad & Precio US\$ & Total US\$ & $\%$ \\
\hline $\begin{array}{l}\text { Cajas EPS } \times 100 \text { vacunas } \\
\text { (poliuretano expandido de } \\
4 \mathrm{~cm} \text { de espesor) }\end{array}$ & 8,0 & 19,3 & 154,0 & 1,93 \\
\hline Geles congelados de $250 \mathrm{~g}$ & 50,0 & 0,7 & 32,5 & 0,41 \\
\hline Geles congelados $500 \mathrm{~g}$ & 50,0 & 0,8 & 37,5 & 0,47 \\
\hline $\begin{array}{l}\text { Cajas de cartón x packs de } \\
5 \text { vacunas para conservar el } \\
\text { producto }\end{array}$ & 10,0 & 1,9 & 19,3 & 0,24 \\
\hline Registradores de temperatura & 16,0 & 5,0 & 80,0 & 1,00 \\
\hline Paños absorbentes & 32,0 & 3,0 & 90,6 & 1,20 \\
\hline Valor de venta del producto & 100,0 & 80,0 & $8.000,0$ & \\
\hline
\end{tabular}

Elaboración propia.

En este caso, el costo del embalaje representa el 5,25\% del precio de venta, que se incluye en las empresas como parte del costo de distribución, variable importante para el área comercial.

\section{ENTRENAMIENTO}

Para mantener el sistema de cadena de frío efectivo y acorde con los estándares establecidos, un factor clave de éxito es contar con personal adecuadamente capacitado y comprometido con la compañía y la sociedad, que tenga en cuenta que no prepara un embalaje sino que lleva salud.

Se recomienda establecer un plan de entrenamiento en cada una de las partes del proceso, esto es definir módulos de entrenamiento teórico y práctico, contar con entrenadores certificados, valoraciones y 
planes de mejoramiento continuo para aquellos colaboradores que no aprobaron el curso, y plan de reentrenamiento. Asimismo, es aconsejable contar con personal backup, y cada vez que ingrese nuevo personal o contratista, darle un entrenamiento básico en aspectos como el plan de contingencia, qué hacer, a quién dirigirse, etcétera.

\section{ANÁLISIS DE RIESGOS}

El análisis de riesgos, realizado mediante cualquiera de sus metodologías, es un punto clave en todo el sistema de gestión de cadena de frío, pues permite:

- Identificar permanentemente los riesgos.

- Definir el grado de severidad de los riesgos.

- Establecer planes de acción e implementar soluciones que permitan reducir la severidad identificada.

Una metodología de análisis de riesgo ampliamente usada es el HAZOP (Hazard and Operabitily Analysis), sistema estructurado técnicamente para identificar y mejorar los riesgos de un equipamiento o de un proceso. El HAZOP se basa en una teoría que supone que los eventos de riesgo son causados por las desviaciones de diseño o de funcionamiento. Está constituido por cuatro actividades principales:

- Definir.- Consiste en definir los objetivos del estudio, el equipo de trabajo y las responsabilidades.

- Preparar.- Trata acerca de la elaboración de un plan de estudios, la recolección de datos, el tiempo de trabajo y la agenda de reuniones.

- Examinar.- Para lo cual hay que dividir el sistema en partes, seleccionar una parte y definir la intención del diseño; identificar la desviación mediante el uso de palabras guía en cada elemento; establecer las consecuencias y las causas; determinar si existe un problema significativo, así como detectar las acciones posibles correctivas o las medidas de mitigación (opcional); acordar otras acciones. Este procedimiento se debe repetir para cada elemento y cada parte del sistema.

- Documentación y seguimiento.- Consiste en revisar lo examinado en el punto anterior; contar con la documentación firmada por los responsables de cada proceso; elaborar el informe del estudio y acordar planes de acción definitivos; elaborar un plan de seguimiento de las 
acciones que se implementarán; repasar el estudio de las piezas del sistema, si fuera necesario; y producir el reporte del resultado final.

Con el análisis de riesgo se logra, entonces, identificar con detalle cada uno de los inconvenientes y minimizarlos o reducirlos en un ámbito de mejora continua de control de los procesos.

\section{CONCLUSIONES Y RECOMENDACIONES}

- La cadena de frío es una especialidad dentro de la cadena de suministro, que garantiza el adecuado abastecimiento de productos en un rango de $2^{\circ} \mathrm{C}$ a $8^{\circ} \mathrm{C}$ para el caso de productos refrigerados.

- El proceso de cadena de frío debe entenderse como un sistema constituido por infraestructura, procesos, tecnologías, plan de entrenamiento y análisis de riesgos, donde el actor más importante es el colaborador de la empresa, el recurso humano.

- No mantener la cadena de frío adecuadamente puede ser causal de pérdida de propiedades de un producto, tales como la potencia y la efectividad para lo que fue desarrollado.

- La cadena de frío no consiste solo en conservar el producto en los almacenes, sino que exige también contar con un sistema de distribución que permita mantenerla adecuadamente; para ello, no menos importantes son los sistemas de embalaje, que pueden representar incluso un 5\% del valor de venta del producto.

- Para una adecuada distribución los perfiles térmicos son clave para el desarrollo de rutas, embalajes y selección del tipo de transporte.

- Para un adecuado manejo de este proceso es muy importante también contar con socios estratégicos, más que proveedores, y promover sinergias que permitan desarrollar tecnología y procesos para mejorar continuamente.

- La tecnología y los procesos van evolucionando, pero el recurso más importante es el ser humano; los colaboradores deben ser entrenados en las empresas, y ser conscientes de que de su entrenamiento, actitud, destreza y comunicación depende la vida de las personas, pues una negligencia puede llevar a la pérdida de las propiedades fisicoquímicas para las que fue diseñado un producto.

- El desarrollo de un sistema de cadena de frío aun cuando tenga la última tecnología, podría tener fallas, dado que el recurso más importante es el humano (colaboradores, socios estratégicos y quienes 
prestan otros servicios). Todos deben estar entrenados adecuadamente, comprometidos con el sistema de cadena de frío, conocer los planes de contingencia y ser conscientes de que juegan un rol preponderante para mantener productos especializados y de alto valor, en las mejores condiciones, hasta llegar al paciente, representado muchas veces por un bebé que recibe una vacuna.

\section{BIBLIOGRAFÍA}

Food and Drugs Administration (1998). "Draft guidance for industry, stability testing of drug substances and drug products". Nueva York: FDA Rockville, MD.

International Conference on Harmonization (noviembre del 2003). "Guidance for industry, Q1A (R2): Stability testing of new drug substances and products". Bruselas.

Irish Medicines Board (marzo del 2006). "Guide to control and monitoring of storage and transportation temperature conditions for medicinal products and active substances". Irlanda: Edition Industrial 003. Versión 01.

USP 27-NF 22 (US Pharmacopeial Convention) (2004). Pharmaceutical dosage form. General Information <1151>. USP, Rockville, MD.

World Health Organization (2002). "Guideline for establishing or improving primary and intermediate vaccines stores". The access to technologies of the department of vaccines and other biologicals. Ginebra: OMS. 